\title{
The key to Injury Prevention would be Daily Stretching for Muscle Flexibility and Strength
}

\author{
${ }^{1}$ Akiboshi Bright Star Training Rehabilitation Center, Kagawa, Japan \\ ${ }^{2}$ Tokushima University / Medical Research, Tokushima, Japan \\ ${ }^{3}$ Japan Masters Athletics, Tokushima Division, Board, Tokushima, Japan \\ ${ }^{4}$ Home-Visit Nursing Station Miki, Kagawa, Japan \\ ${ }^{5}$ Japan Masters Athletics, Kagawa division, Vice-President, Kagawa, Japan
}

Hiroya Hanabusa ${ }^{1}$, Akito Moriyasu ${ }^{1}$, Hiroshi Bando ${ }^{2},{ }^{3 *}$, Makoto Takasugi $^{4}$, Mitsuru Murakami ${ }^{5}$

DOI: 10.36348/jaspe.2021.v04i01.002

| Received: 10.01.2021 | Accepted: 22.01.2021 | Published: 23.01.2021

*Corresponding author: Hiroshi Bando

\section{Abstract}

Authors' and collaborators have continued research concerning stretching, the flexibility of thoracic spine. In this study, author himself continued our stretching protocol for 4 months. The changes were i) the spreading degree of open legs, ii) bridge posture bending the whole body backward, iii) finger floor distance (FFD) with 3 to $18 \mathrm{~cm}$. The purpose was selfverification by habituation training, and clinical efficacy was found. For other effects, he has not been injured for the period as soccer player at all, and has felt comfortable operation subjectively. This process will be beneficial for managing various patients and athletes from now.

Keywords: finger floor distance (FFD), Muscle Flexibility, sports, orthopedic problems.

Copyright () 2021 The Author(s): This is an open-access article distributed under the terms of the Creative Commons Attribution 4.0 International License (CC BY-NC 4.0) which permits unrestricted use, distribution, and reproduction in any medium for non-commercial use provided the original author and source are credited.

\section{INTRODUCTION}

Human can perform a variety of movements, which are from adequate smooth behaviors of the trunk and extremities. Our trunk consists of basically three parts including thorax, spine and pelvis [1]. Furthermore, all kinds of joints are involved in our smooth movements, such as facet, costal and sacroiliac joints [2]. Then, flexibility is an important factor for satisfactory function [3]. When we want to move freely as we like, some training for stretching would be necessary, especially in subjects on some sports and patients with orthopedic problems [4].

In recent years, the authors' group has continued medical practice and research on sports orthopedics, rehabilitation, educational seminar and workshop for various subjects with all ages $[5,6]$. Among them, there were some reports concerning stretching, the flexibility of thoracic spine and spinal column [7]. In the case of Masters athletics, highimpact running styles are prone to injury, and we have reported recommendations for biaxial and push running styles [8].

The first author himself has been a soccer player from elementary school, and has suffered from constant physical injuries for long years. For every situation, his coaches and managers often pointed out the necessity for stretching exercise. Regarding our recent work for sports clinic and rehabilitation, there are actually many requests from various subjects to "improve flexibility to prevent injuries" [9]. It seems to be important that to teach and make them understood the mechanism of stiff body, injury and adequate stretching method [10].

In order to indicate persuasive stretch methods, the author has actually tried to make a habit of stretching and training. Consequently, this article presents the key points of injury and stretching.

\section{Protocol}

1. Purpose of this intervention trial: The purpose of this intervention is self-verification by habituation training [11]. The training contents in this trial are classified into two aspects. One is mainly stretching to increase flexibility, and the other is strength training to increase stability [12]. In addition, we conducted some trainings to improve physical manipulation. For this situation, two specific goals were speculated as follows: i) to indicate a model movement for teaching athletes with one's own body, and ii) to change the physical body that was often injured as the result 
of voluntary training.

2. Training implementation content manual: The content of the training is the stretching and training methods that our rehabilitation group has traditionally continued and performed so far. There are several textbooks by Moriyasu et al., [13]. "Habituation training" was conducted by the standard method. Regarding stretching, we have focused more on "frequency rather than one time" and carried out using the leisure time.

3. Three tests to compare: Three tests were compared before and after the intervention to determine the effectiveness of stretching training. The three methods are i) open legs and compare the degree of spread of bilateral legs, ii) perform a bridge posture bending the whole body backward and compare the degree, iii) evaluate Finger Floor Distance (FFD) with upper body forward with the hand on the floor. These three tests were conducted for comparing the degree of stretching and bending back and forth.

\section{RESULT}

Along the protocol, the intervention was conducted and three markers were compared between 0 month and 4 months. The changes in these were as follows (Figure-1). i. Open legs: When comparing before and after, the degree of open legs seems to be increased in the latter. Essentially, this assessment must meet three criteria: a) with the elbows on the floor, b) with the toes facing forward, and c) with the knees not bent. The posture at 0 month did not satisfy the element a) and b) due to the lack of flexibility, and then it is difficult to make a strict comparison. The factors a-c) were met in the posture at 4 months, which can be used as an evaluation standard.

ii. Bridge posture: After the intervention, the elbows were no longer flexed with stretched straight, the distance between the hands and feet was narrowed, and the floor-to-umbilical height was increased. These showed the increased flexibility.

iii. Finger Floor Distance (FFD): The change in FFD was from $+3 \mathrm{~cm}$ to $+18 \mathrm{~cm}$, showing an improvement in flexibility.

Another beneficial effect was present, where the author himself has not been injured at all in this season for playing soccer and futsal. Furthermore, he realized that he felt his complete and easy operation of the body as the same level as he was a younger soccer player before, which was from subjective impression.

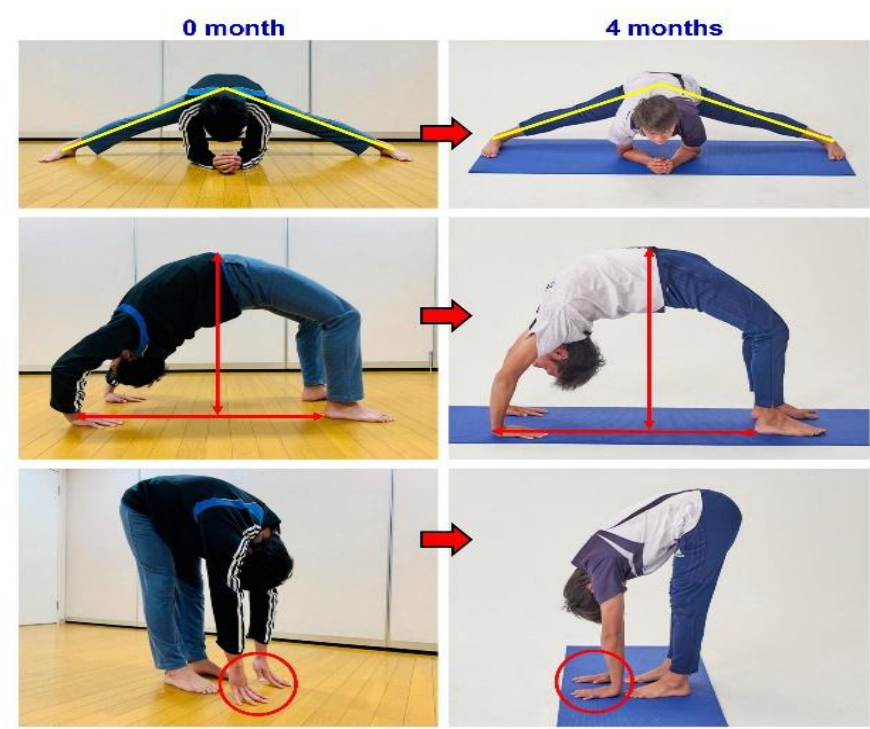

Fig-1: The changes in the flexibility after the intervention trial of Upper: open legs, middle: bridge posture, low: FFD

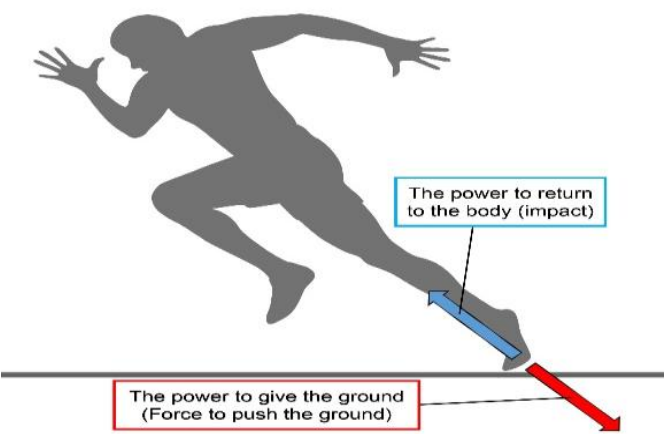

Fig-2: The impact power from the ground in the case of short distance sprinting

\section{DISCUSSION}

Various sports injuries are seen in the field of sports clinical practice. Of these, the "strong impact" applied to the body is thought to be the cause in many cases. An example can be shown in athletics sprints [14]. Strong power is applied to the ground and the repulsive force returned to the body is used to move forward (Fig-2). The stronger the force returned to the body, the stronger the impact on the body and the greater the risk of injury. Thus, if the shock absorption cannot disperse, strong stress may accumulate in weak or unstable parts of the body, leading to injuries and pain such as tissue damage [15]. It is the role of 
flexibility to absorb and disperse this strong impact. Greater flexibility helps to prevent various injuries.

Two functions are important in exercise and sports movements, which are strength and flexibility [16]. The former maintains stability to support the body and exerts power for exercise. The latter keeps mobility for smooth movement and determines the range of motion. The balance between them has been indispensable. If one side is increased, high performance cannot be achieved and the burden on the body increases.

For example, even a strong rugby player with low flexibility can move less and increase the risk of joint injury [17]. Even a highly flexible rhythmic gymnast can be injured by excessive movement if the muscles supporting the body are insufficient. From the above, combining flexibility and muscle strength seems to be a necessary condition for injury prevention.

Human body has a tendency of narrowing the range of motion of muscles and joints that are not used on a daily basis [18]. In other words, if one always uses joints, one can move them smoothly and maintain high flexibility. Conversely, if one uses joints infrequently, the joints will show less flexibility. Thus, it is important to increase the frequency of stretching every day and make it a habit for greater flexibility [19]. For example, it is easier to improve flexibility by stretching for 10 minutes $\times 1$ times or 5 minutes $\times 12$ times, rather than stretching for 60 minutes $\mathrm{x} 1$ time a day.

There are various cases in the clinical setting. Even if one continues stretching every day, one can see that the flexibility does not improve easily [20]. One of the reasons for this would be that muscles with rather tense and stiff for long are difficult to stretch and contract smoothly. In other words, muscles that are highly tense and unable to stretch or contract are less responsive. In such cases, start by relaxing the muscles. If the muscle with tension can be vibrated to some degree, it may gradually loosen and become easier to stretch [21].

This report has its limits. The subject is the author himself and is a report of only one case. There are few evaluation items, and then this report does not provide a certain perspective. It is expected that the physical therapist has conducted a trial associated with beneficial experience for positive management for patient support. We will make the most advantage of current experience for our medical activities in the future [22].

In summary, this verification reaffirmed the importance of flexibility and strength. Furthermore, this experience can bring continuous daily stretching since then. Several injuries are difficult to prevent completely, but can be reduced as much as possible. In order to prevent injuries, it is important to practice stretching and muscle training for long. This article will be hopefully one of the references for developing future research.

Conflict of Interest: The authors declare no conflict of interest.

Funding: There was no funding received for this paper.

\section{REFERENCES}

1. Narimani, M., \& Arjmand, N. (2018). Threedimensional primary and coupled range of motions and movement coordination of the pelvis, lumbar and thoracic spine in standing posture using inertial tracking device. Journal of Biomechanics, 69, 169-174.

2. Manchikanti, L., Schultz, D. M., Benyamin, R. M., \& Falco, F. J. E. (2018). Thoracic Facet Joint Interventions. In: Manchikanti, L., Kaye, A., Falco, F., \& Hirsch, J. (eds). Essentials of Interventional Techniques in Managing Chronic Pain. Springer, Cham.

3. Donti, O., Gaspari, V., Papia, K., Panidi, I., Donti, A., \& Bogdanis, G. C. (2020). Acute Effects of Intermittent and Continuous Static Stretching on Hip Flexion Angle in Athletes with Varying Flexibility Training Background. Sports, 8(3), 2836.

4. Kang, M. H., \& Oh, J. S. (2020). Effects of selfstretching with mobilization on shoulder range of motion in individuals with glenohumeral internal rotation deficits: a randomized controlled trial. Journal of Shoulder and Elbow Surgery, 29(1), 36-43.

5. Moriyasu, A., Bando, H., Murakami, M., Inoue, T., Taichi, A., Wakimoto, K., ... \& Akayama, R. (2018). Pole exercise causes body changes in physical flexibility and exercise function. Journal of Novel Physiotherapies, 8(1), 377.

6. Bando, H., Murakami, M., \& Moriyasu, A. (2019). Spinal Mobility with Concept of the Combination of Motion Spheres in the Body. Neurophysiology and Rehabilitation, 2(1), 51-52.

7. Kurihara, R., Fujimoto, D., Dakeshita, T., Moriyasu, A., \& Bando, H. (2019). The influence of Pole exercise on the range of motion of thoracic spine. Clinical Research in Orthopaedics, 2(1):1-5.

8. Murakami, M., \& Bando, H. (2019). Same directionality of foot straight line and forward movement. ARC Journal of Research in Sports Medicine, 4(1), 14-16.

9. Moriyasu, A. Akiboshi Bright Star training rehabilitation center. https://akiboshi.jp

10. Bando, H., Murakami, M., \& Moriyasu, A. (2020). Adequate treatment of nutrition and exercise for elderly people associated with sarcopenia and nutritional disorder. International Medicine, 2(3), 162-164.

11. Djenovic, V. D. (2017). Methodology of training 
young football. College of professional studies "football academy Belgrade" Belgrade. College of Professional Studies. pdf (d1wqtxts1xzle7.cloudfront.net)

12. Mortazavi, F., \& Nadian-Ghomsheh, A. (2018). Stability of Kinect for range of motion analysis in static stretching exercises. PloS one, 13(7), $\mathrm{e} 0200992$.

13. Moriyasu, A., Murakami, M., \& Bando, H. (2020). Aiming for operability by habit training in the baseball and all sports. International Physical Medicine \& Rehabilitation Journal, 5(2), 89-91.

14. Beato, M., Bigby, A. E., De Keijzer, K. L., Nakamura, F. Y., Coratella, G., \& McErlainNaylor, S. A. (2019). Post-activation potentiation effect of eccentric overload and traditional weightlifting exercise on jumping and sprinting performance in male athletes. PLoS One, 14(9), e0222466.

15. Crow, J., Semciw, A., Couch, J., \& Pizzari, T. (2020). Does a recent hamstring muscle injury affect the timing of muscle activation during high speed overground running in professional Australian Football players?. Physical Therapy in Sport. 43:188-194.

16. Lee, C. L., Chu, I. H., Lyu, B. J., Chang, W. D., \& Chang, N. J. (2018). Comparison of vibration rolling, nonvibration rolling, and static stretching as a warm-up exercise on flexibility, joint proprioception, muscle strength, and balance in young adults. Journal of sports sciences, 36(22), 2575-2582.

17. Sever, O., \& Zorba, E. (2018). Comparison of effect of static and dynamic core exercises on speed and agility performance in soccer players. Isokinetics and Exercise Science, 26(1), 29-36.

18. Zhou, W. S., Lin, J. H., Chen, S. C., \& Chien, K. Y. (2019). Effects of dynamic stretching with different loads on hip joint range of motion in the elderly. Journal of sports science \& medicine, 18(1), 52-57.

19. Donti, O., Papia, K., Toubekis, A., Donti, A., Sands, W. A., \& Bogdanis, G. C. (2018). Flexibility training in preadolescent female athletes: Acute and long-term effects of intermittent and continuous static stretching. Journal of sports sciences, 36(13), 1453-1460.

20. Heshmatipour, M., Esfandiari, A., Kazemi Naeini, M., Raei, M., Firoozpur, O., Shariatinia, N., \& Hushmandi, K. (2019). Effect of Active Dynamic Versus Passive Static Stretching on Hamstring Muscle Tightness in Healthy Female Students: A Randomized Trial Study. Hospital Practices and Research, 4(4), 134-138.

21. Azuma, N., \& Someya, F. (2020). Injury prevention effects of stretching exercise intervention by physical therapists in male high school soccer players. Scandinavian Journal of Medicine \& Science in Sports, 30(11), 2178 2192.

22. Bando, H., Moriyasu, A., \& Murakami, M. (2020). Effective Practice Using Pole Exercise Would Contribute for The Situation of "Exercise is Medicine". Journal of Advances in Sports and Physical Education, 3(8), 132-134. 